\title{
Design and Experiment of a Hand Movement Device Driven by Shape Memory Alloy Wires
}

\author{
Yangwei Wang, Shufang Zheng, Jingquan Pang, Sanping Li, and Jian Li $\mathbb{D}$ \\ College of Mechanical and Electrical Engineering, Northeast Forestry University, Harbin 150040, China \\ Correspondence should be addressed to Jian Li; lijian@nefu.edu.cn
}

Received 13 October 2020; Revised 23 February 2021; Accepted 17 March 2021; Published 31 March 2021

Academic Editor: Weitian Wang

Copyright (c) 2021 Yangwei Wang et al. This is an open access article distributed under the Creative Commons Attribution License, which permits unrestricted use, distribution, and reproduction in any medium, provided the original work is properly cited.

\begin{abstract}
Hand is one of the most flexible and useful organs of the human body. However, cases of hand function loss due to diseases and accidents abound, which brings a lot of inconvenience to patients' lives. This paper introduced a flexible hand movement device (HMD) driven by shape memory alloy (SMA) wires, which is compact, lightweight, and easy to use during activities of daily living (ADL). Firstly, we elaborated the design and modelling process, including the driving analysis of SMA wires. Secondly, the manufacturing process and testing method of the HMD are presented. Thirdly, the results of the experiment are discussed in detail. Through the experiment, we obtained the bending angle and operating space of fingers. Moreover, the joint angle as well as the fingertip force can be controlled using PWM. A maximum angular movement of $45^{\circ}, 62^{\circ}$, and $71^{\circ}$ can be generated at the metacarpophalangeal (MCP), proximal interphalangeal (PIP), and distal interphalangeal joints (DIP) of a finger using an SMA wire, respectively. Then, the operating space of the entire hand can be calculated based on the experimental results. Besides, an estimated $40 \mathrm{~N}$ of grasping force can be produced for a whole hand. In conclusion, this research indicates that the finger movement driven by the HMD is similar to a healthy person, which can meet the needs of ADL.
\end{abstract}

\section{Introduction}

Kinds of neural diseases, such as stroke, may result in the loss of hand function, which causes enormous damage to patients' physical and mental health [1-3]. Many studies have confirmed that rehabilitation therapy can limit stroke complications and facilitate brain remodeling [4-6]. Thus, researchers have designed and fabricated a variety of robots to assist rehabilitation progress. Primordially, Wege and Conti proposed some kinematic chain mechanism for hand exoskeletons $[7,8]$. But the rigid structures in the linkage mechanisms usually impede the therapeutic potential of robotics, which causes joint alignment issues and secondary joint damage [9].

To combat these shortcomings, cable-driven mechanisms, pneumatic actuators, and artificial muscles are rapidly utilized as an alternative. Under Chu and Patterson, most hand rehabilitation devices use pneumatic systems or cable systems as actuators [9]. Hyunki In et al. designed a wearable robot called the Exo-Glove with a soft tendon routing system. The fingers were pulled by Bowden cable actuated by motors. Lucas et al. [10] proposed an EMGcontrolled pneumatic cable-driving hand exoskeleton in 2004. It was lightweight, and adjustable, but inconvenient to carry around because of the bulgy pneumatic pistons and other affiliate devices.

With the development of novel artificial muscles, pneumatic artificial muscle (PAM), twisted and coiled polymer (TCP) muscles, and SMA are increasingly being used in prosthetics and rehabilitation as actuators.

PAM has been widely used in the industrial and scientific field due to its high strength and small weight. However, it exhibits a highly nonlinear relationship between contraction and pulling force, which makes it difficult to model and control [11]. Besides, commonly used PAM actuators are relatively bulky and take up a lot of space, which is not convenient to install and carry around. Lokesh et al. proposed iGrab [12] in 2017, which was powered by TCP muscles. The device is lightweight but the output force still needs improvement. A study by Matheus et al. benchmarked 
grasping and manipulating forces and found that most objects of daily living weigh less than $1.5 \mathrm{Kg}$ [13]. In terms of the research conducted by $\mathrm{Wu}$ et al. [14], the maximum tensile force of the TCP muscle is only $4.5 \mathrm{~N}$, which is quite low compared to high stress [15] SMA ( $\varnothing 0.3 \mathrm{~mm}, 40 \mathrm{~N})$. Besides, the total diameter of the coiled TCP muscles is $1.24 \sim 1.41 \mathrm{~mm}( \pm 0.02)$, which is thicker than SMA wires $(0.3 \mathrm{~mm})$. ASR glove [16] was proposed by Alireza et al. in 2017; it was actuated by SMA and could provide a grasping force of more than $40 \mathrm{~N}$, which was sufficient for ADLs. However, the joint angles still need improvement.

In general, there are some terminologies which generally completes the power criteria of different actuators. However, there is no universal standard for measuring actuators. The judging criteria are depending on what the author uses the actuator for [17]. In [18], the trade-off between force output and the actuation speed is the main issue in rehabilitation. Compared to TCP muscles, SMA wires can exert higher stress. Apart from this, the pseudoelasticity and shape memory effect (SME) of SMA are very useful in neurology and neuromuscular rehabilitation applications [18]. The SME enables the SMA to integrate the sensor with actuator which can simplify the structure. Moreover, by exploiting the hysteretic properties, we can control and dampen unwanted involuntary movements during the actuation process to improve stability [18]. But there are a few studies that used SMA as actuators for hand rehabilitation because it is difficult to design a soft and pliable exoskeleton to fit well with SMA wires and the hand. Furthermore, the limited strain of the SMA wires stops it from being used as actuators [19]. To overcome this shortcoming, we designed a novel range extender mechanism to magnify the limited strain. Above all, it is very urgent to study the anatomical structure of human hands and design a new flexible exoskeleton structure driven by SMA wires. However, the current research on the new structure and design method is not innovative enough.

Based on the abovementioned design, we came up with an idea to actuate the finger in a way that is flexible and lightweight. As is known to us all, the pulley mechanism can be used to enlarge the displacement, so it can enlarge the shrinkage of the SMA wires. However, because the SMA wire has high hardness and does not fit well with the pulley, we use ropes instead. To connect the ropes and SMA wires, we designed some connection mechanisms. Therefore, 3D printed range extenders which consist of the structures mentioned above are designed to amplify the displacement of the SMA wire. To increase the energy conversion rate, we wrap the SMA wires in the insulation tube to reduce the heat radiation. The rest of the paper is organized as follows: the structural design and model establishment is elaborated. Then, the manufacturing process and testing methods are listed. The results of the experiments and the discussion are included in the results and discussion.

\section{Structural Design and Model Establishment}

For human hands, each finger can be viewed as a serial open kinematic chain, which consists of three joints and links. The
MCP joint has two rotational degrees of freedom (DoF), and the PIP and DIP joints have one DoF each, as shown in Figure 1 . To simplify the modelling process, the MCP joint is regarded as a rotating joint with only one rotational DoF. Therefore, each finger can be considered as a three-revolute joint robotic manipulator, as shown in Figure 1.

According to the different starting points of the muscle, the muscles can be divided into internal and external muscles. The internal muscle is mainly responsible for finetuning the balance of the joint. And the external muscle (FDP, FDS, and EDC) is the power source of finger motion. During finger flexion, the contraction of the FDP drives the movement of the joints together with internal muscles. During finger extension, the contraction of EDC contracts against the passive resistance of the FDP, which makes three finger joints rotate synchronically to complete finger extension [20]. The schematic diagram of the finger muscles is shown in Figure 2.

To better simulate the motion of the human fingers, the structure of the finger exoskeleton should mimic the biological structure. SMA, which have a shape memory effect, are widely used as artificial muscle actuators for bionic devices. By applying heat, SMA wires can be shortened through phase transformation, which can take the place of human muscles to activate the finger flexion and extension.

But there is an important limitation of SMA wires: low strain rate compared to its initial length which requires longer wires. To overcome this shortcoming, a novel range extender mechanism is designed to enlarge the driving displacement of SMA wires, as shown in Figure 3.

A pully block consists of two fixed pullies and three free pullies are used to enlarge the stroke of the SMA wire to six times the original (as seen in Figure 3(c)) which is long enough to actuate the finger. In this way, the HMD is more compact and lightweight, and its portability and comfort level is improved, compared with the ASR glove [16]. Besides, PE lines are used for directly pulling the finger to abduct and adduct. It can withstand large tensile forces without breaking. And it is tight and wear-resistant, has good plasticity, and will not get tangled up. In this way, the effectiveness of the device is well guaranteed. The rope on the dorsal side and palmar side of the finger is equivalent to the EDC and FDP, respectively, which imitate the physiological structure of the human hand.

The schematic diagram of the hierarchical structure in the glove is shown in Figure 4. The range extenders are stuck between two layers of textile by adhesive. The distal end of the range extender is connected to the fingerstall fixed on the fingertip through PE lines; the other end is fixed at a wiring ring at the elbow through the SMA wire. The activation of each finger needs two SMA wires (one for the flexion, and the other one for extension); thus 10 SMA wires are required for a hand, as shown in Figure 5.

By applying a voltage to two ends of the SMA wire, it thermally contracted and then the pullies were pulled in the range extender. Thereby, the PE line is actuated, and then the finger is pulled to flexion and extension. Due to the physiological structure of the finger, when we pull the distal 


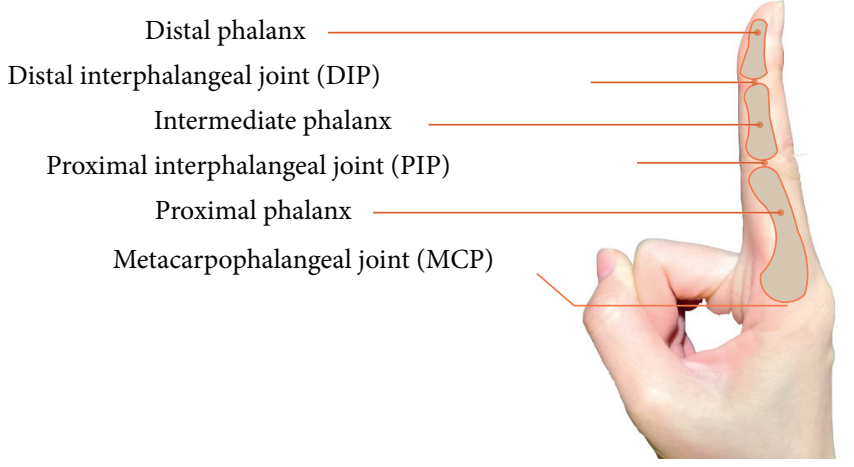

(a)

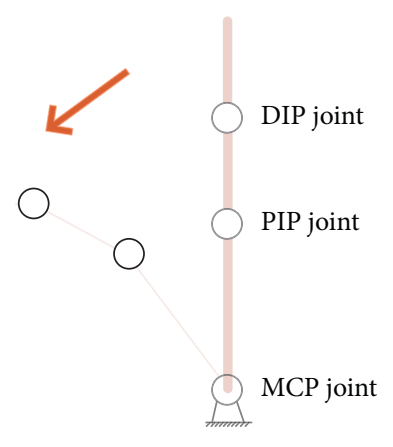

(b)

FIgure 1: Theoretical model. (a) Names of different finger joints. (b) Simplified model.

(a)
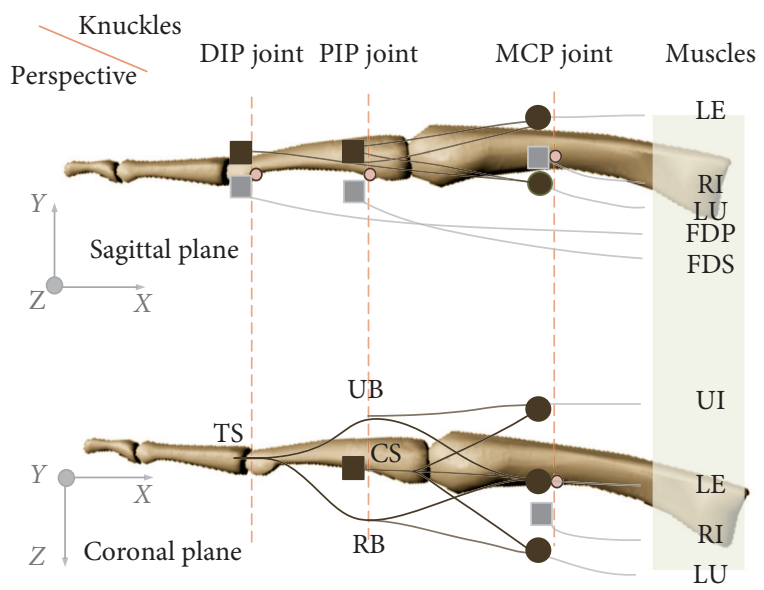

Figure 2: Theoretical model of the extensor mechanism of the finger. (a) Sagittal plane. (b) Coronal plane.

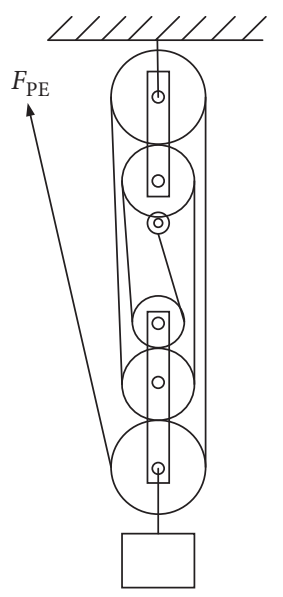

(a)

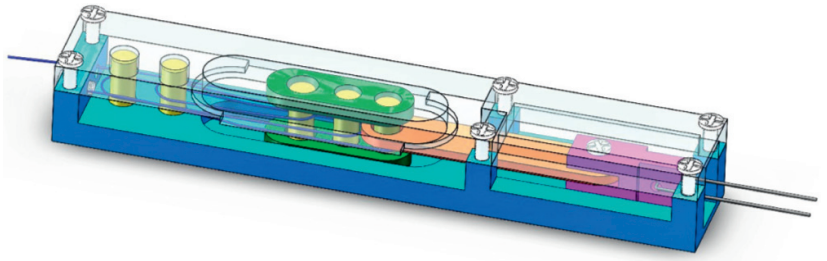

(b)

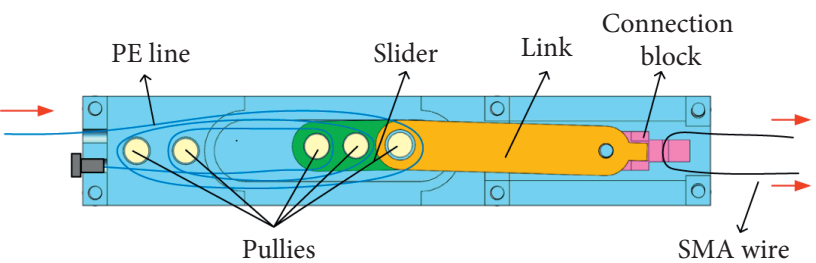

(c)

Figure 3: Schematic diagram of the range extender. (a) Schematic diagram of the pulley mechanism. (b) 3D view. (c) Section view. 


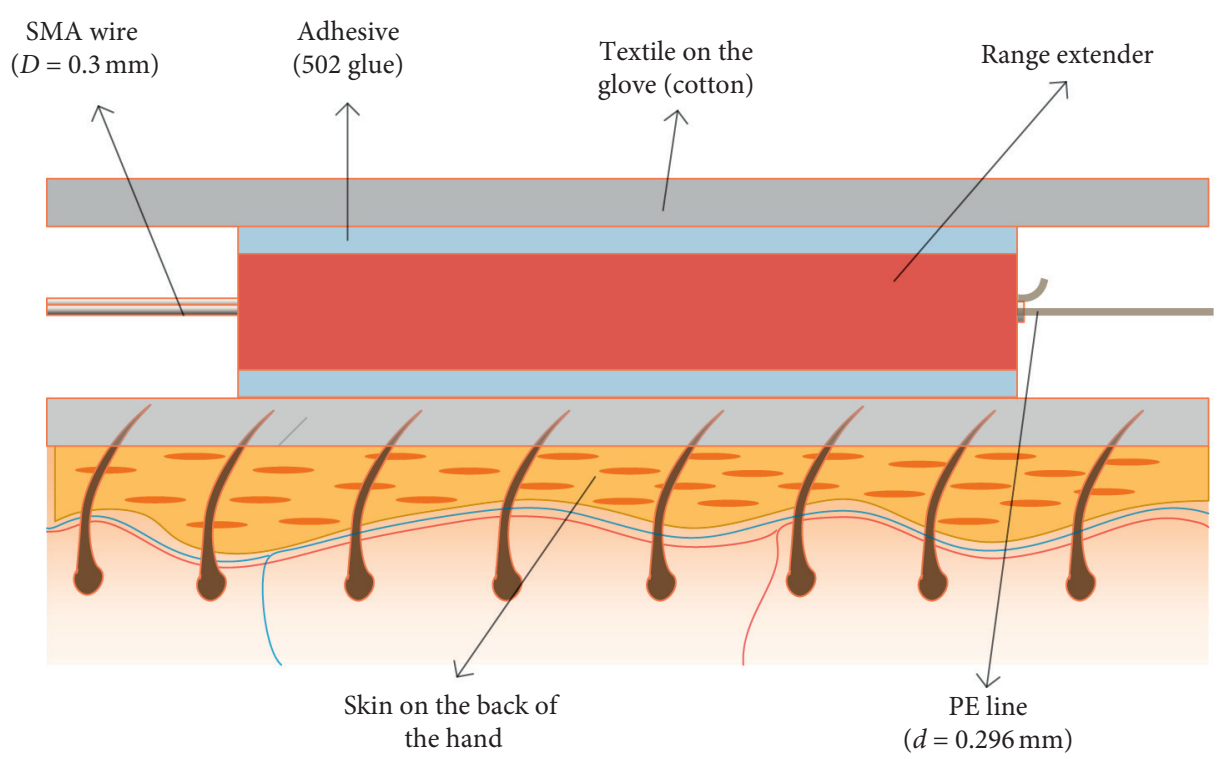

Figure 4: Schematic diagram of the hierarchical structure.

phalanx of the finger, the three joints will rotate synchronously.

The relationship between tendon displacement and joint angle is articulated in this section. As shown in Figure 6, we built a bidimensional model of the fingers on the sagittal plane, and the fingers are modeled as a 3-link kinematic chain. The tendon displacement during flexion can be extracted from joint angles:

$$
x=l_{1} \sin \left(\frac{\theta_{1}}{2}\right)+l_{2} \sin \left(\frac{\theta_{2}}{2}\right)+l_{3} \sin \left(\frac{\theta_{3}}{2}\right),
$$

where $x$ represents the tendon displacement $(\mathrm{mm}), l_{1}, l_{2}$, and $l_{3}$ are the thickness of the MCP, PIP, and DIP joints (mm), respectively. $\theta_{1}, \theta_{2}$, and $\theta_{3}$ are joint angle $\left(^{\circ}\right)$ of MCP, PIP, and DIP joints, respectively.

Dividing the $x$ obtained above by the strain rate can get the theoretical length of SMA wires; then because of the pulley mechanism inside the range extender, the length required is 6 times shorter. Besides, since we fold the SMA wires in half, the actual length is then doubled:

$$
L_{\min }^{\text {Act }}=\frac{2 x}{\mu \varepsilon},
$$

where $\mu$ represents the shrinkage of the SMA wires, $\varepsilon$ represents the magnification of the shrinkage of the SMA wire by the range extender, and $L_{\min }^{\mathrm{Act}}$ is the minimum actual length of the alloy wire for a single finger.

SMA wire is a novel flexible actuator. Both the shape memory effect and the superelasticity arise from martensitic transformations [21]. During the heating process, when the temperature reaches a specific value, the martensitic crystal phase is transformed into austenite crystal phase, which is stable at higher temperature, and the structure becomes more compact and therefore the SMA wire contracts to its original length and produces shrinkage stress. Therefore, it can be used to actuate the finger motion.
Power can be applied to both ends of the SMA wire through a power supply. Because of the heating effect of the current, the temperature rises, and then the phase transformation occurs. Thus, the temperature is very important for the activation process. The relationship between the temperature and the applied voltage is shown as follows:

$$
m_{\mathrm{SMA}} c_{p} \frac{d T(t)}{d t}=\frac{U^{2}}{R} \tau-h A\left(T(t)-T_{0}\right),
$$

which can be simplified as

$$
\frac{d T}{d t}=\frac{U^{2} \tau}{c \rho_{\mathrm{SMA}} l^{2} \rho_{R}}-\frac{2\left[h_{1}+h_{2}\left(T-T_{0}\right)\right]\left(T-T_{0}\right)}{c \rho_{\mathrm{SMA}} r_{\mathrm{SMA}}},
$$

where $m_{\mathrm{SMA}}=\rho_{\mathrm{SMA}} \pi r_{\mathrm{SMA}}^{2} l_{\mathrm{SMA}}$ is the weight of the SMA wire, $r_{\mathrm{SMA}}$ is the density of the SMA wire, $l_{\mathrm{SMA}}$ is the length, $c_{p}$ is the specific heat, $T$ is the temperature, $t$ is time, $U$ is the voltage, $R=\rho_{R} l_{\mathrm{SMA}} / \pi r_{\mathrm{SMA}}^{2}$ is the resistance of the SMA wires, $\rho_{R}$ is the resistivity, $\tau$ is the PWM ratio, $h$ is the heat convection coefficient, $A=2 \pi r_{\mathrm{SMA}} l_{\mathrm{SMA}}$ is the heat transfer area of the SMA wire, and $T_{0}$ is the ambient temperature. The relationship of heat convection coefficient temperature is a linear function, according to [22].

$$
h=h_{1}+h_{2}\left(T-T_{0}\right) \text {, }
$$

where $h_{1}$ is baseline heat transfer coefficient and $h_{2}$ is correction heat transfer coefficient. According to [23], the phase transformation during heating can be described as

$$
\sigma-\sigma_{0}=D(\eta) \varepsilon-D\left(\eta_{0}\right) \varepsilon_{0}+\Omega(\eta) \eta-\Omega\left(\eta_{0}\right) \eta_{0}+\Theta\left(T-T_{0}\right),
$$

where $\sigma$ is the terminal stress when the transformation is over, $\sigma_{0}$ is the initial stress, $D=\left(D_{m}-D_{a}\right) \cdot \eta+D_{a}$ is the elastic modulus, $D_{m}$ and $D_{a}$ are the elastic modulus of martensite and Austenite, respectively, $\eta$ is the shape memory factor (between 0 and 1), $\varepsilon_{0}$ is the initial strain, $\Theta$ is 


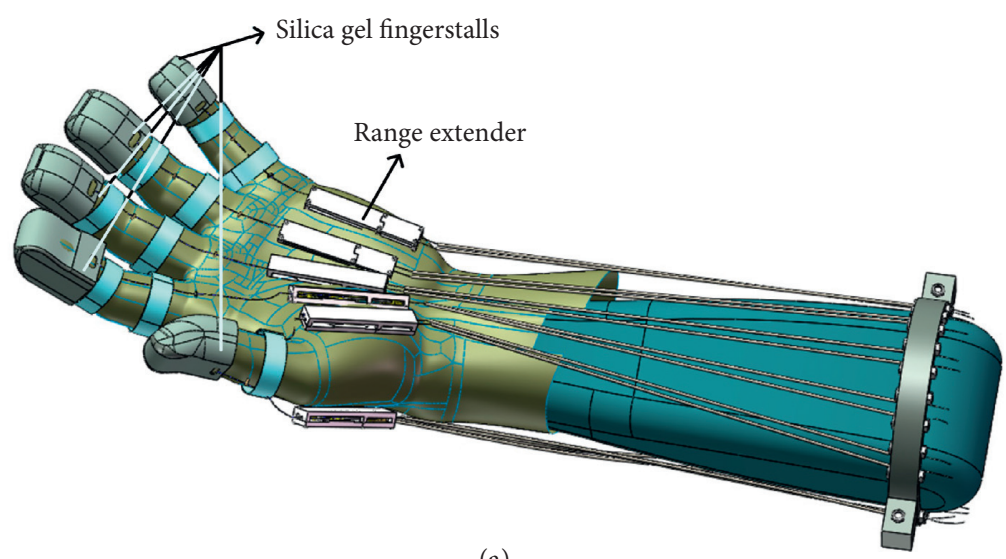

(a)

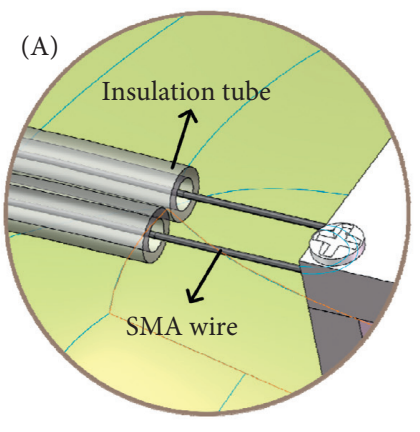

(b)

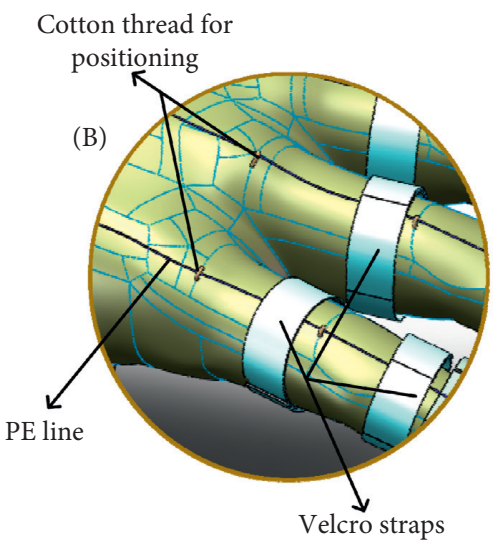

(c)

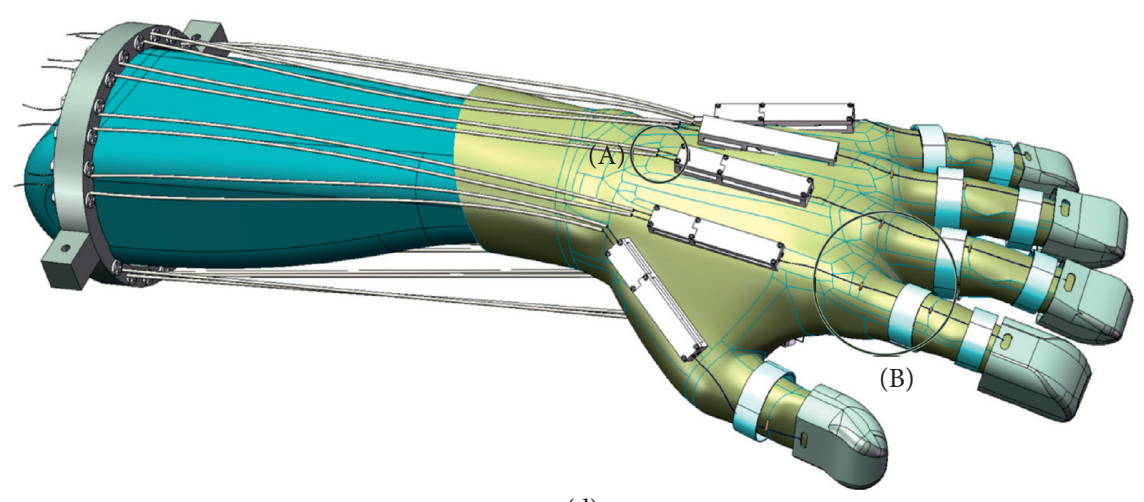

(d)

Figure 5: Conceptual design of the HMD.

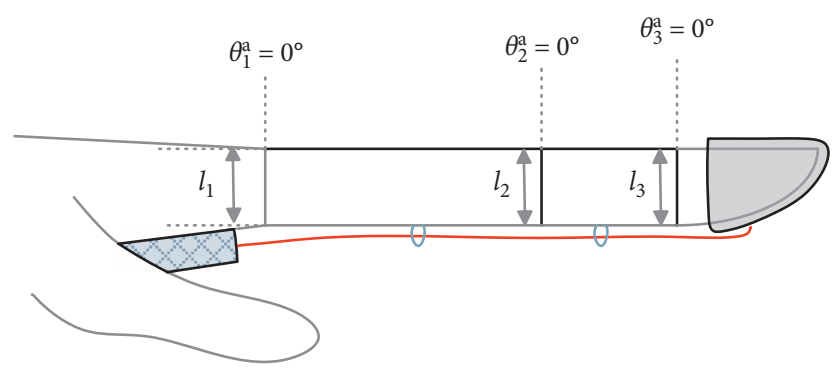

(a)

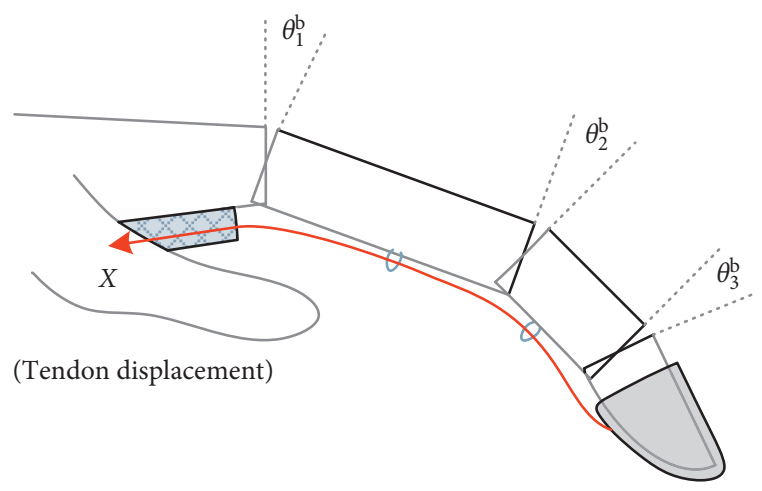

(b)

Figure 6: Relationship between tendon displacement and joint angle: (a) untensioned tendon and (b) tensioned tendon. 
the thermoelastic coefficient, $\Omega(\eta)=-\varepsilon_{L} D(\eta)$ is the shape memory modulus, and $\varepsilon_{L}$ is the maximum residual strain of the SMA wire. The performance parameters of SMA wires are shown in Table 1.

\section{Prototype Manufacturing and Testing Methods}

The prototype of the HMD is shown in Figure 7. The SMA wires used in this experiment are from Dongguan Baohong Metal Material Co., Ltd. (shop570466789.taobao.com), which is $0.3 \mathrm{~mm}$ in diameter. To fabricate the HMD, we used a pair of cotton gloves as the base. The base of the range extender is 3D printed using resin (made from Somos ${ }^{\circledR} \mathrm{GP}$ Plus, http://www.dsmsomos.com) and assembled by bolts. Outside the range extender, the PE lines (Camilo flagship store, Dyneema) are fixed on the cotton glove by Velcro tapes (VELCRO ${ }^{\circledR}$ Brand Fasteners). As we know, the cross section shape of the forearm is not a regular circle. To better fit the shape of the arm, we use flexible materials to act as wiring rings. The wiring ring which is fixed near the elbow is 3D printed using flexible TPU. It consists of two halves, connected by bolts. An insulating tube is used to block the heat on the SMA wires during the actuation and prevent it from scalding the skin.

Reference [20] gives the range of motion of each finger joint, seen in Table 2. Table 3 depicts the thickness of the knuckles of a 21-year-old woman. Substituting the data in Tables 2 and 3 into equation (1) gives the length of the SMA wires that we need. Considering the errors in the experiment, the SMA wires that we used are $600 \mathrm{~mm}$ long. Therefore, the effective length of the SMA wire is $300 \mathrm{~mm}$ because we folded them in half. Assuming that the strain rate of the SMA wire is $4 \%$, the theoretical displacement of the PE lines when activated is $72 \mathrm{~mm}$.

To verify the driving effect of the HMD, we designed several experiments to study the relationship between the finger bending angle over time and applied voltage. The operating space of fingers is also calculated. To control the fingers to reach different joint angles, we used PWM pulse to activate the SMA wires, and the maximum fingertip force during the process is measured by a pressure sensor.

A DC power supply is used to drive the fingers to bend, each SMA wire passed through a current of $1 \mathrm{~A}$. A highspeed camera is used to record the bending angle of the joints under different driving voltages. After analyzing the data, we got graphs of the angles with time and the relationship between them. We measured the length of each knuckle, as shown in Table 4. Subsequently, the operating space of the fingertip can be obtained.

We also recorded the fingertip force using a pressure transducer to see if the device can generate enough grip strength. So we activate the fingers under different voltages, and the fingertip forces can be seen on the PC. The experimental setup is shown in Figure 8.

Mechanically, the final design encompassed a system that includes a DC power supply (IT6722 A $80 \mathrm{~V} / 20 \mathrm{~A} /$ $400 \mathrm{~W}$ from ITECH Electronics Co., Ltd.), HMD, pressure sensor (ZNBSQ-V $24 \mathrm{~V}$ 0 10 V Bengbu Zhongnuo Sensor
TABle 1: Performance parameters of SMA wire.

\begin{tabular}{lcc}
\hline Parameter & Description & Value \\
\hline$\rho_{\text {SMA }}$ & Density of SMA $\left(\mathrm{kg} / \mathrm{m}^{3}\right)$ & $6.5 \times 10^{3}$ \\
$r_{\text {SMA }}$ & Diameter of SMA $(\mathrm{mm})$ & 0.3 \\
$l_{\text {SMA }}$ & Length of SMA $(\mathrm{mm})$ & 600 \\
$c_{p}$ & Specific heat $\left(\mathrm{J} /\left(\mathrm{Kg} \times{ }^{\circ} \mathrm{C}\right)\right)$ & 870 \\
$\rho_{R}$ & Resistivity $(\Omega \cdot \mathrm{m})$ & $1.11 \times 10^{-6}$ \\
$h_{1}$ & Baseline heat transfer coefficient & 1100 \\
$h_{2}$ & Correction heat transfer coefficient & 19 \\
$M_{f}$ & Martensite final temperature $\left({ }^{\circ} \mathrm{C}\right)$ & 34.5 \\
$M_{s}$ & Martensite start temperature $\left({ }^{\circ} \mathrm{C}\right)$ & 61.1 \\
$A_{s}$ & Austenite start temperature $\left({ }^{\circ} \mathrm{C}\right)$ & 69.3 \\
$A_{f}$ & Austenite final temperature $\left({ }^{\circ} \mathrm{C}\right)$ & 109.5 \\
\hline
\end{tabular}

Co. Ltd.), and PC (as shown in Figure 9). On the premise of satisfying the requirements, the smaller the current, the longer the service life. Besides, at high heating rates $\left(>100^{\circ} \mathrm{C} / \mathrm{min}\right)$, the stress increases with a significant decrease in strain [24]. Therefore, we use a current of $1 \mathrm{~A}$ to activate the SMA wires. The experimental results show that it can meet the requirements of the driving frequency of the HMD in reference [10].

\section{Results and Discussion}

4.1. Finger Bending Test. We can get a series of pictures from the videos that we recorded. For the sake of brevity, only the index finger is discussed in detail, as shown in Figure 9. It can be seen that the device can perform about 6 rehabilitation exercises per minute. We meet the requirement of [25], five times per minute. Besides, compared to 3 or 4 times a minute in [26], we have greatly improved on this basis.

Figure 10 shows the process of flexion of the fingers at different voltages. Since the voltages we used are higher than the voltage threshold to reach the phase transformation temperature, therefore, the maximum joint angle of fingers is the same and has nothing to do with the applied voltages. But we only recorded the joint angles in the first ten seconds after the voltage excitation for simplification. We can see that when we tune up the voltage, the movement will also accelerate. To this end, more rehabilitation exercises can be performed if we increase the voltage value in the experiment.

Figure 11 shows the comparison between the theoretical joint angle and the experimental joint angle of the DIP joint over time under different driving voltage. We can see that the experimental joint angles are consistent with the theoretical angle.

Dr. Wenrui Chen used principal component analysis to analyze the three joints of a single finger and found that the three joint angles of a finger can be expressed linearly by a principal component vector [27]. The relationship among the finger joint angles is shown in equation (7).

$$
P_{1}=\left(p_{1}^{1} p_{1}^{2} p_{1}^{3}\right)^{T}=\left(\begin{array}{lll}
0.610 .700 .37
\end{array}\right)^{T} .
$$

Therefore, when one of the joints rotates at a certain angle, the motion of other joints can be determined accordingly. For this experiment, the average value of the maximum joint angle of our joints at different voltages is 


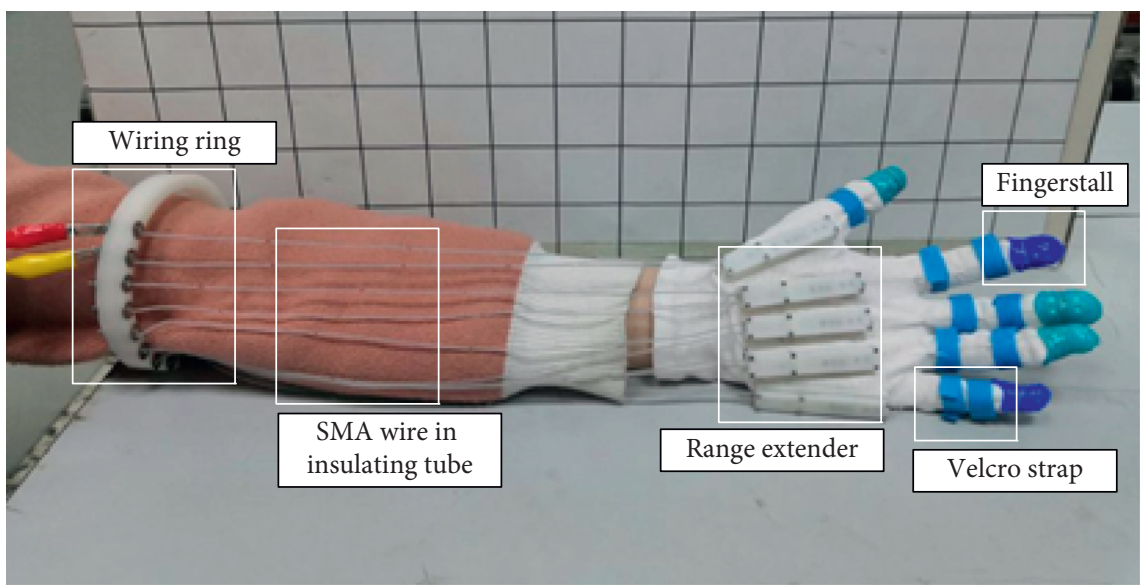

Figure 7: Prototype of the HMD.

TABLE 2: Range of variation of each finger joint.

\begin{tabular}{lccc}
\hline Joints & MCP & PIP & DIP \\
\hline Range of motion $\left(^{\circ}\right)$ & $0 \sim 85$ & $0 \sim 100$ & $0 \sim 80$ \\
\hline
\end{tabular}

TABLE 3: Thickness of the knuckles of a 21-year-old woman (index finger).

\begin{tabular}{lcccc}
\hline Index finger & MCP & PIP & DIP \\
\hline Thickness $(\mathrm{mm})$ & 24 & 15 & 12 \\
\hline
\end{tabular}

TABLE 4: Length of each phalanx.

\begin{tabular}{|c|c|c|c|c|c|}
\hline Phalanx (mm) & Thumb & Index finger & Middle finger & Ring finger & Little finger \\
\hline Distal phalanx & 26 & 24 & 23 & 23 & 24 \\
\hline Intermediate phalanx & 34 & 24 & 25 & 25 & 15 \\
\hline Proximal phalanx & 30 & 44 & 50 & 47 & 42 \\
\hline
\end{tabular}

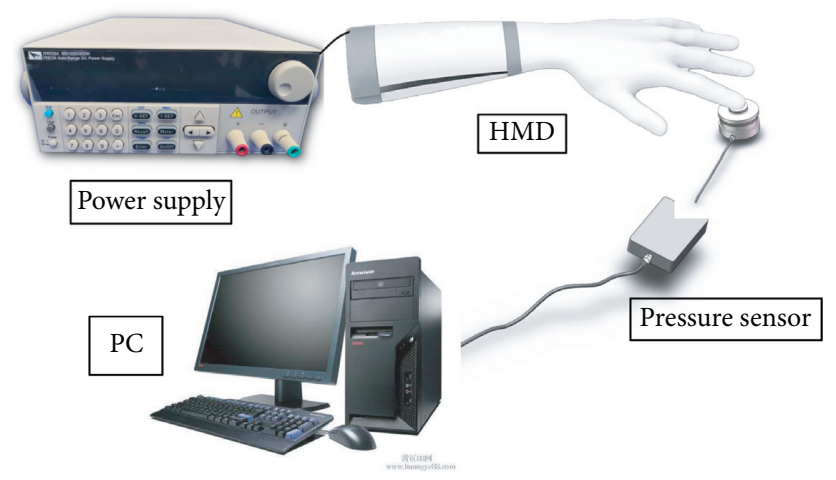

FIGURE 8: Experimental platform for HMD.

shown in Table 5. The maximum joint angle of each joint of the finger during the experiment (regardless of the voltage value) is recorded in Table 6.

The joint angles of the MCP, PIP, and DIP joints are related to each other, showing a proportional relationship among them in this experiment (Table 5), which is similar to the ratio obtained in [27], that is,

$$
\theta_{1}: \theta_{2}: \theta_{3}=30.08: 42.15: 29.3
$$

and the experiment showed a capacity of activating the index finger to adduct. The current system is an underdriven system; the theoretical joint angles can be obtained based on the tendon displacement. But some experimental errors cause the shrinkage of the SMA wire to be wasted. For 

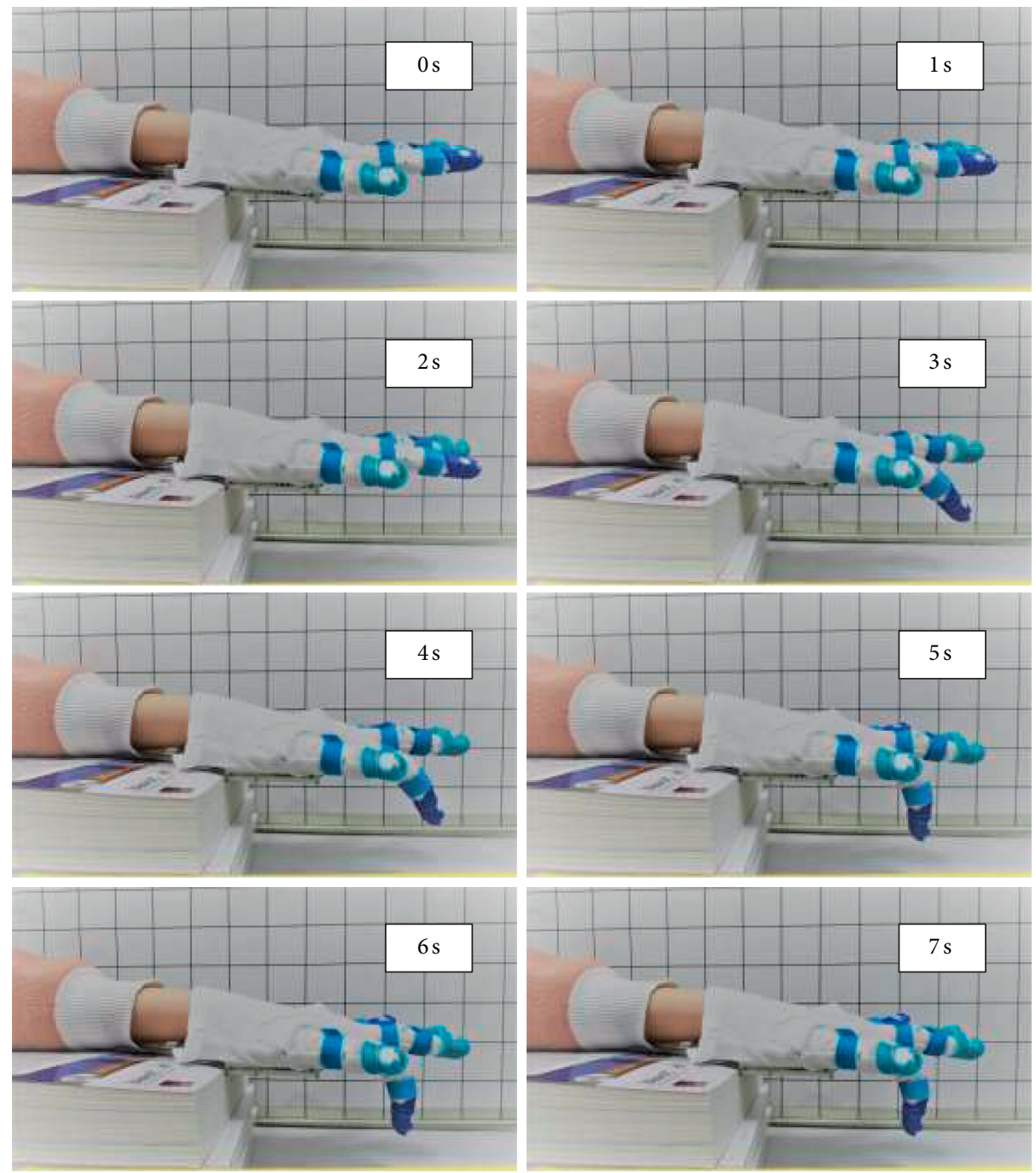

FIGURE 9: Index finger postures after actuation for $1 \mathrm{~s}$ time intervals.

example, due to the slack of the SMA wire and PE lines as well as the irregular shape of the arm, the tendons cannot always fit well with the surface of the arm. Besides, the shrinkage of the SMA wires is constantly changing with time and voltage. Besides, the friction in the mechanism also needs to be taken into consideration. In conclusion, the actual displacement of the PE line is different from the theoretical calculation. Therefore, we assume that only a quarter of the displacement generated by SMA wires is in effect. That is, the shrinkage of the PE line is $18 \mathrm{~mm}$.

Putting equation (8) and the thickness of the index finger (Table 3) into equation (1), we can get the following theoretical angular displacement of each finger joint:

$$
\theta_{1}=37.25^{\circ}, \theta_{2}=52.20^{\circ}, \theta_{3}=36.28^{\circ} \text {. }
$$

The experimental angular displacement of the three finger joints in the index finger is shown as follows:

$$
\theta_{1}=34.48^{\circ}, \theta_{2}=53.95^{\circ}, \theta_{3}=28.98^{\circ} \text {. }
$$

We can see that there are some deviations between the experimental joint angles and the theoretical ones; this is because of the individual differences in various persons. On the whole, the theoretical joint angles are almost consistent with the experimental one, which indicates the feasibility of our design.

\section{Operating Space When Wearing HMD}

We can get the operating space at the end of the finger through Tables 4 and 5. By simplifying the framework of the fingers, we built a link coordinate frame for each joint, as shown in Figure 12, and subsequently built the kinematics of the manipulator based on the Denavit-Hartenberg (D-H) convention. The $\mathrm{D}-\mathrm{H}$ parameter table of the finger is shown in Table 7.

Bring the joint angle in Table 5 into the link coordinate frame (Figure 12, Table 7); the results are shown in Figure 13, which shows the achievable flexion/extension ranges of five fingers when wearing the HMD. Then we can get the size of the object that can be grasped when wearing the device. The blue bands in Figure 13 represent the track set of fingertip movement trajectories, which is also the operating space at 


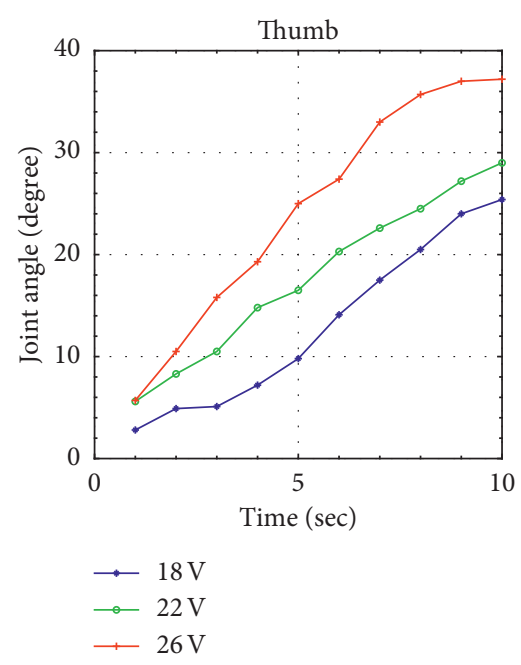

(a)

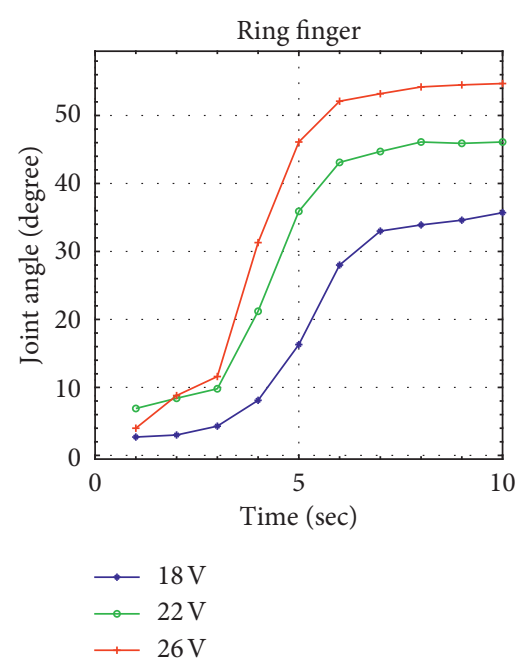

(d)
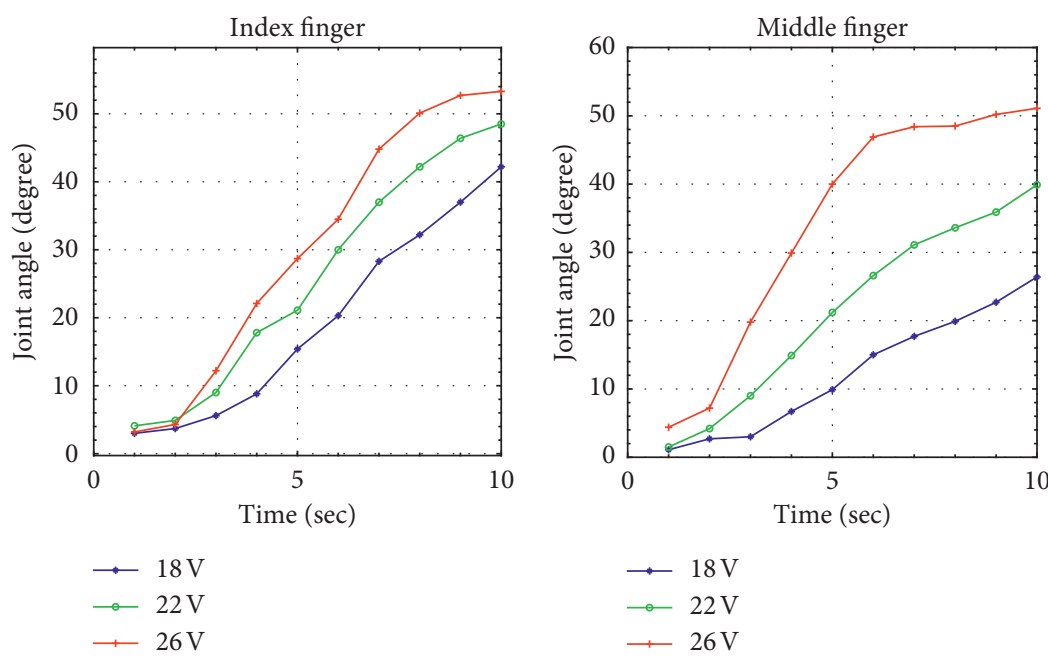

(b)

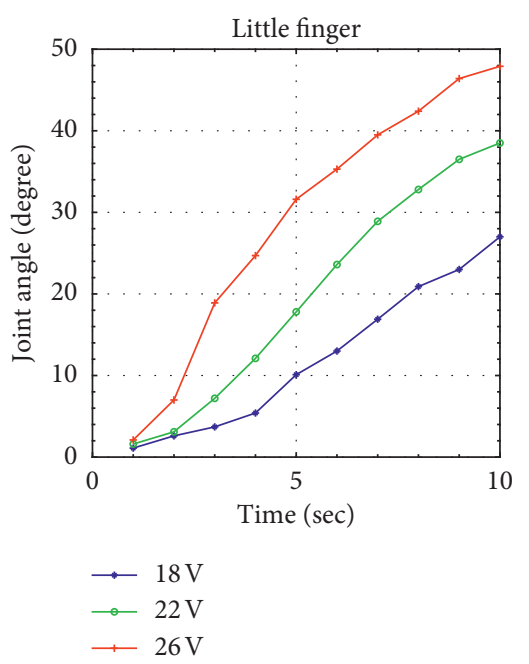

(e)

FIGURE 10: Relationship between joint angle and time of different fingers.

the end of the finger. The pink dots indicate three finger joints (DIP, PIP, and MCP joints). The three short rods represent the three phalanxes, respectively (blue for proximal phalanx and middle phalanx, the red one for the distal phalanx). They are all described in the sagittal plane. Figure 14(b) shows the posture of the hand when it is fully opened. Its original state is shown in Figure 14(a).

We found that the minimum radius of the sphere that can be grasped is $16 \mathrm{~mm}$, which is about the diameter of a small ring, as shown in Figure 14(b). The subject can pinch thin slices of any thickness using HMD, like a piece of paper. Also, the patients can turn on and off the device easily due to the flexible wearable structure. From Table 6, It can also be found that the joint angles in this experiment have been improved compared to Lokesh's [12]. Besides, because of the novel range extender, we can use fewer SMA wires to achieve the same joint angle compared with Alireza's [16]. This has brought a significant reduction in costs. At the same time, the device uses a driving method that mimics human muscle, and there are no protruding parts like reference $[10,28]$ did.
Thus, the whole device is more lightweight, compact, and portable, which is more applicable to ADL.

\section{PWM Control of Flexion Angle of HMD}

To achieve the specific hand posture, we need to be able to control the finger joint angle to a certain degree. Through tuning the PWM power ratio, we can activate the finger joint angle to a certain degree. The experimental setup is shown in Figure 15.

Take the index finger as an example; we control the sum of the DIP, PIP, and MCP joint angles to a certain degree and stabilize for several seconds under different PWM pulse duty cycle; the relationship between the theoretical analysis and experimental results is shown in Figure 16 . We can see that, through tuning the duty cycle of the PWM pulse, the HMD can activate the fingers to reach different joint angles and can be stable for a while. The higher the PWM duty cycle, the larger the stabilized joint angle can be reached. 


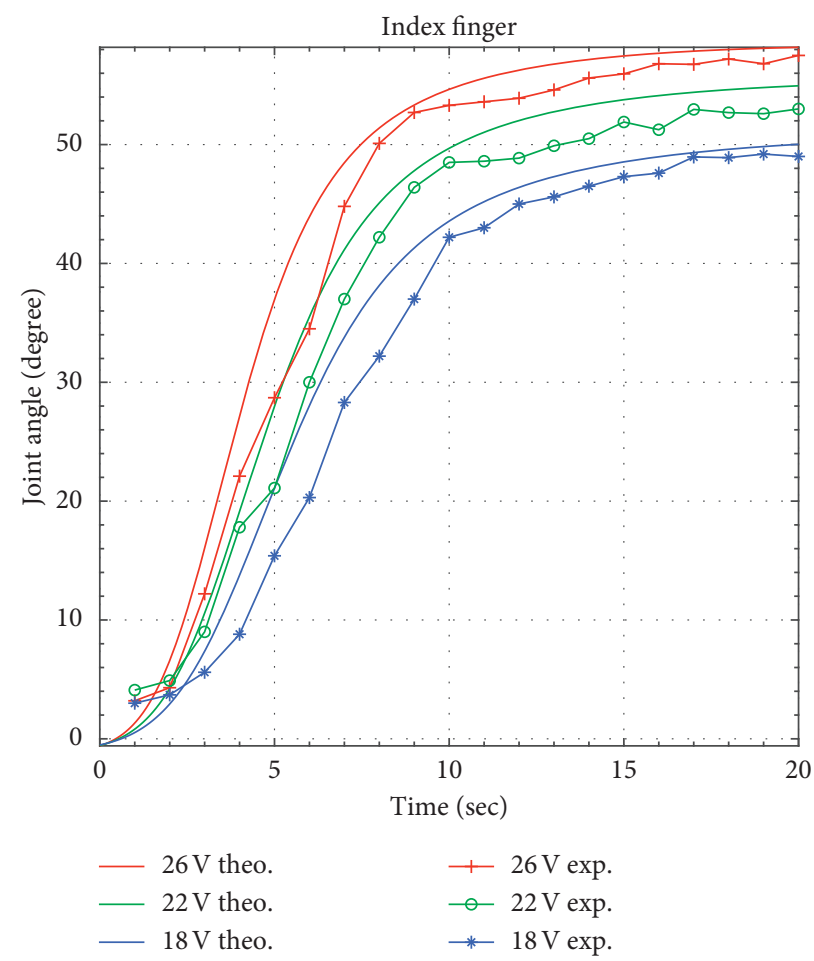

FIgURE 11: Variations of DIP Joint angle over time (index finger, experiment versus theory).

TABLe 5: Average value of the maximum joint angle of our joints at different voltages $\left({ }^{\circ}\right)$.

\begin{tabular}{lcccccc}
\hline Joint & Thumb & Index & Middle & Ring & Little & Average \\
\hline MCP & 22.1 & 36.9 & 27.4 & 26.3 & 37.7 & 30.1 \\
PIP & 6.9 & 54.4 & 57.7 & 49.8 & 42.3 & 42.2 \\
DIP & 40.7 & 28.9 & 26.7 & 25.6 & 24.6 & 29.3 \\
\hline
\end{tabular}

TABle 6: Angular maximum displacement for each joint of all five fingers $\left({ }^{\circ}\right)$.

\begin{tabular}{lccccc}
\hline Joint & Thumb & Index & Middle & Ring & Little \\
\hline MCP & 25.1 & 39.7 & 28.1 & 29.0 & 44.2 \\
PIP & 15.0 & 58.3 & 62.2 & 57.6 & 48.4 \\
DIP & 71.6 & 33.3 & 38.9 & 42.1 & 27.3 \\
\hline
\end{tabular}

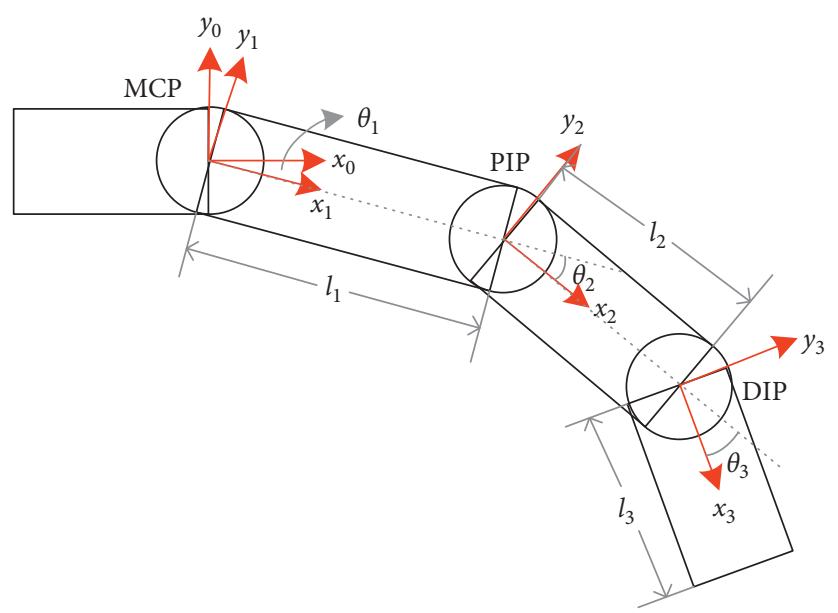

Figure 12: Coordinate system of a single finger $\left(l_{1}, l_{2}, l_{3}\right.$ denote the length of the proximal phalanx, intermediate phalanx, and distal phalanx, respectively. $\theta_{i}$ is the joint angle of MCP, PIP, and DIP joint $\left.(i=1,2,3)\right)$. 
TABLE 7: D-H parameters.

\begin{tabular}{lcccc}
\hline Phalanx & $a_{i-1}$ & $\alpha_{i-1}$ & $d_{i}$ & $\theta_{i}$ \\
\hline (1) Proximal phalanx & 0 & 0 & 0 & $\theta_{1}$ \\
(2) Intermediate phalanx & $l_{1}$ & 0 & 0 & $\theta_{2}$ \\
(3) Distal phalanx & $l_{2}$ & 0 & 0 & $\theta_{3}$ \\
\hline
\end{tabular}

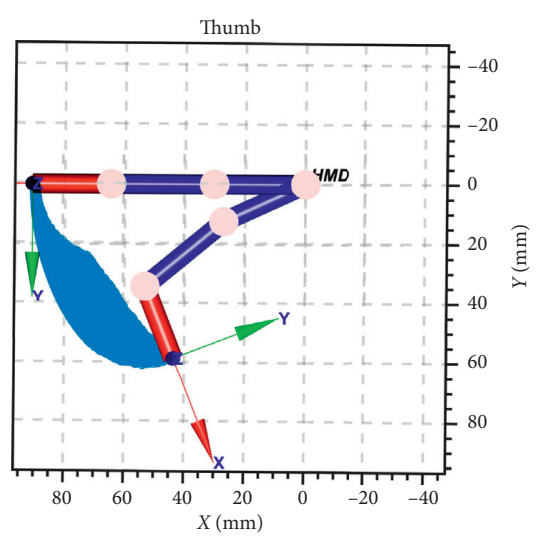

(a)

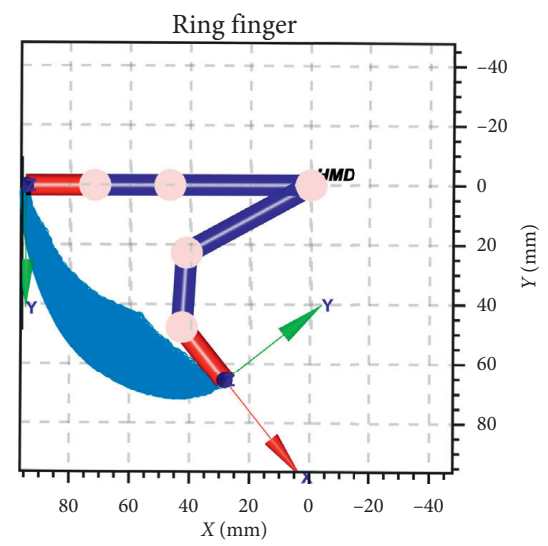

(d)

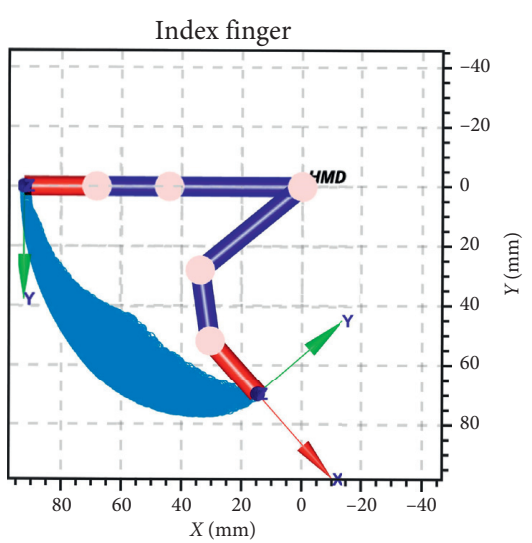

(b)

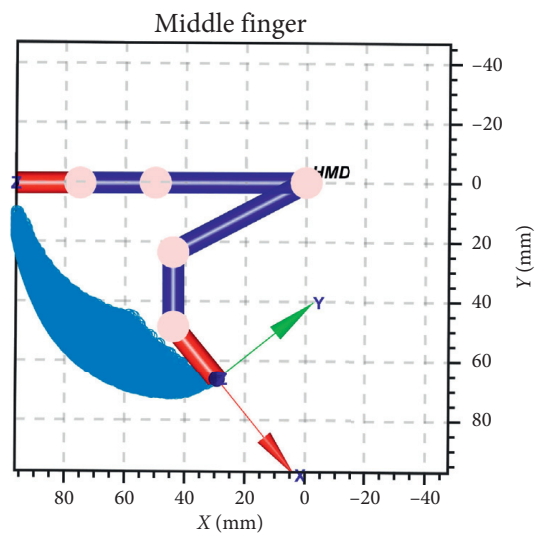

(c)

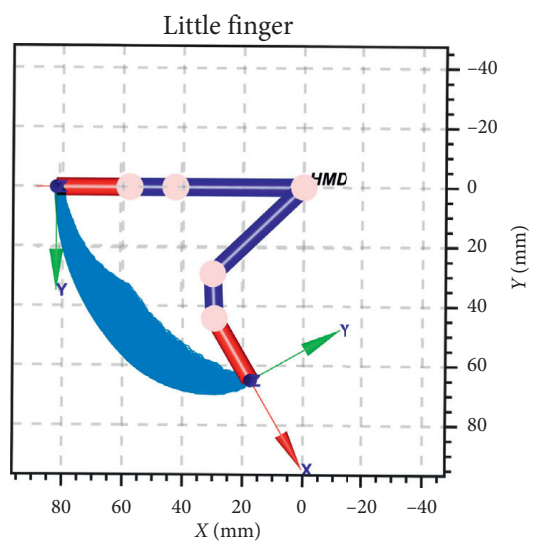

(e)

Figure 13: Visualization of the operating space of each finger. (a) Thumb. (b) Index finger. (c) Middle finger. (d) Ring finger. (e) Little finger.

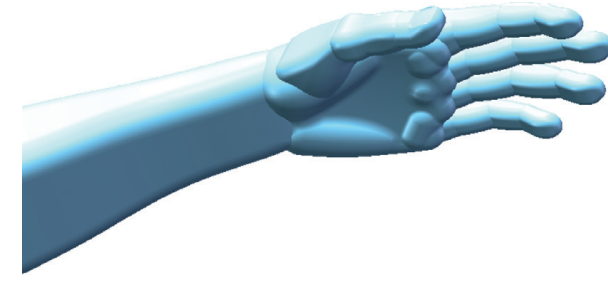

(a)

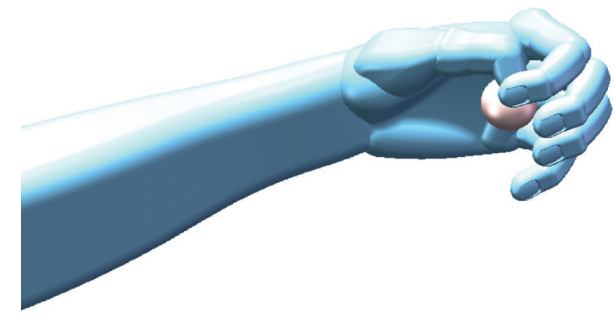

(b)

FIGURE 14: Schematic diagram of the operating space of the hand.

6.1. The Fingertip Force after Wearing the HMD. The fingertip force is shown in Table 8. In the experiment, we used a pressure sensor to measure the fingertip forces. The results are as follows.
According to Table 8, the average fingertip force generated by each finger is about $8 \mathrm{~N}$. Compared with the grip strength of $26.2 \mathrm{~N}$ produced by the five fingers when using 


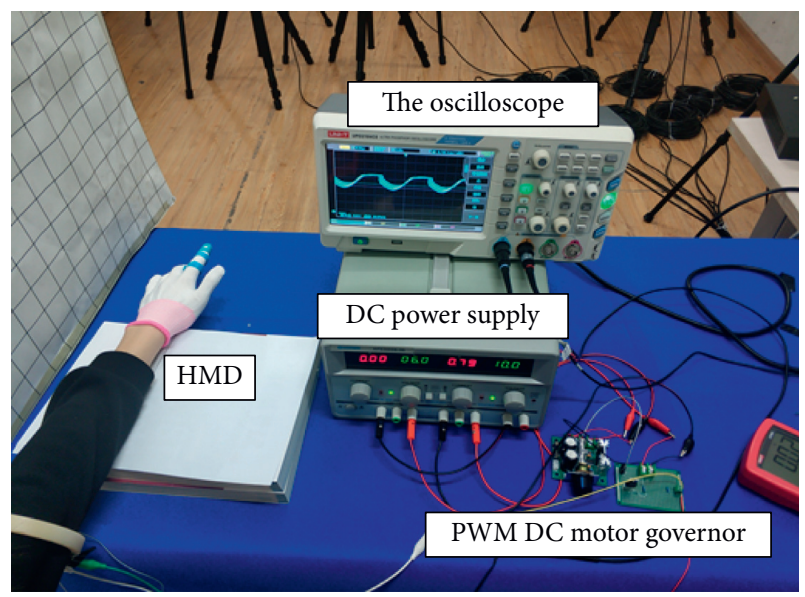

FIGURE 15: Experimental setup of PWM control of flexion angle of HMD.
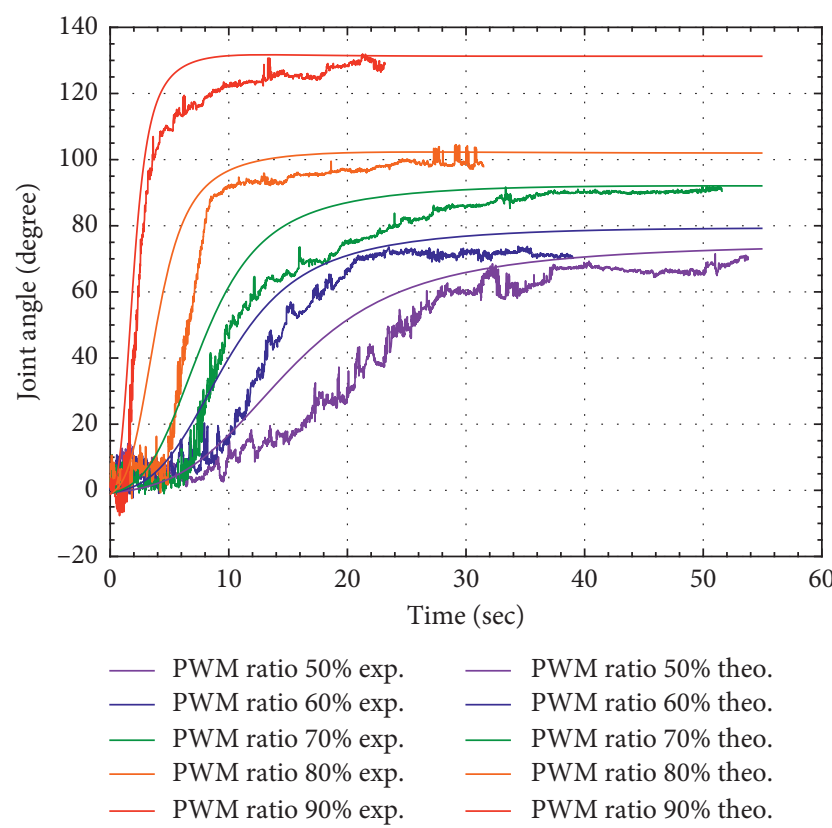

Figure 16: Total joint angle under different PWM ratio.

TABLe 8: Fingertip force for each finger actuated by SMA wires.

\begin{tabular}{lccccc}
\hline & Thumb & $\begin{array}{c}\text { Index } \\
\text { finger }\end{array}$ & $\begin{array}{c}\text { Middle } \\
\text { finger }\end{array}$ & $\begin{array}{c}\text { Ring } \\
\text { finger }\end{array}$ & $\begin{array}{c}\text { Little } \\
\text { finger }\end{array}$ \\
\hline Force $(\mathrm{N})$ & 10 & 6 & 8 & 10 & 6 \\
\hline
\end{tabular}

the HandSOME in reference [28], we have increased the fingertip force by $52.7 \%$, which is capable of most ADL.

\section{Conclusions}

In this study, we proposed a hand movement device actuated by SMA wires. The suggested soft robotic glove uses a tendon-driven system and 3D-printed novel range extenders. The structure of the range extender and the HMD is explained in detail. Besides, we also experimentally verified that the voltage has a positive correlation with the speed of finger movement. The joint angles actuated by the proposed HMD are also sufficient for ADL and can be controlled to a certain degree through tuning the duty cycle of PWM pulse. This device can provide reference values for other related research and can help the ADL of patients with hand defects. It can approximately generate a fingertip force of about $8 \mathrm{~N}$, which is sufficient for most patients. The robustness and life cycle of the SMA wires as well as the numbers of SMA wires used will be investigated during fatigue tests in the future to improve the practical performance of the robot. And we will also work on how to improve the frequency of the device to further improve the rehabilitation process.

\section{Data Availability}

The data used to support the findings of this study are included within the supplementary information files.

\section{Conflicts of Interest}

The authors declare that there are no conflicts of interest regarding the publication of this paper.

\section{Acknowledgments}

This project was supported by the National Natural Science Foundation of China (52075089), the Natural Science Foundation of Heilongjiang Province (LH2019E008), and the Fundamental Research Funds for the Central Universities (2572018BF03).

\section{Supplementary Materials}

Video S1: video of fingers flexing. (Supplementary Materials)

\section{References}

[1] C. Calautti and J.-C. Baron, "Functional neuroimaging studies of motor recovery after stroke in adults," Stroke, vol. 34, no. 6, pp. 1553-1566, 2003.

[2] G. Kwakkel, B. J. Kollen, and H. I. Krebs, "Effects of robotassisted therapy on upper limb recovery after stroke: a systematic review," Neurorehabilitation and Neural Repair, vol. 22, no. 2, pp. 111-121, 2008.

[3] S. B. Cassandra, S. Z. Britt, and J. B. Heidi, "Structural plasticity in adulthood with motor learning and stroke rehabilitation," Annual Review of Neuroscience, vol. 41, pp. 25-40, 2018.

[4] X. X. Lou, Active Rehabilitation for Stroke Patient Based on Cortico-Muscular Coherence, Zhejiang University, Hangzhou, China, 2014.

[5] X. B. Gao, Research on Experiment and Evaluation of Rehabilitation Training Robot, Chongqing University, Chongqing, China, 2008.

[6] Y. M. Wang, "Observation on the curative effect of early rehabilitation treatment in stroke patients with hemiplegia," Chinese Journal of Practical Nervous Diseases, vol. 20, no. 9, pp. 54-56, 2017.

[7] A. Wege and G. Hommel, "Development and control of a hand exoskeleton for rehabilitation of hand injuries," 2005. 
[8] R. Conti, E. Meli, and A. Ridolfi, "A novel kinematic architecture for portable hand exoskeletons," Mechatronics, vol. 35, pp. 192-207, 2016.

[9] C. Y. Chu and M. Patterson, "Soft robotic devices for hand rehabilitation and assistance: a narrative review," Journal of NeuroEngineering and Rehabilitation, vol. 15, no. 1, pp. 1-14, 2018.

[10] L. Lucas, M. DiCicco, M. DiCicco, and Y. Matsuoka, "An EMG-controlled hand exoskeleton for natural pinching," Journal of Robotics and Mechatronics, vol. 16, no. 5, pp. 482-488, 2004.

[11] K. C. Wickramatunge and T. Leephakpreeda, "Study on mechanical behaviors of pneumatic artificial muscle," International Journal of Engineering Science, vol. 48, no. 2, pp. 188-198, 2010.

[12] L. Saharan, M. J. de Andrade, W. Saleem et al., "iGrab: hand orthosis powered by twisted and coiled polymer muscles," Smart Materials and Structures, vol. 26, no. 10, 2017.

[13] K. Matheus and A. M. Dollar, "Benchmarking grasping and manipulation: properties of the objects of daily living," 2010.

[14] L. J. Wu, M. J. de Andrade, L. K. Saharan et al., "Compact and low-cost humanoid hand powered by nylon artificial muscles," Bioinspiration \& Biomimetics, vol. 12, no. 2, pp. 1-16, 2017.

[15] Y. L. Wang, Interfacial Mechanical Properties of Shape Memory Alloy Composite, Harbin Engineering University, Harbin, China, 2011.

[16] A. Hadi, K. Alipour, S. Kazeminasab, and M. Elahinia, "ASR glove: a wearable glove for hand assistance and rehabilitation using shape memory alloys," Journal of Intelligent Material Systems and Structures, vol. 29, no. 8, pp. 1575-1585, 2018.

[17] B. Tondu, Artificial Muscles for Humanoid Robots, Humanoid Robots, Human-Like Machines, I-Tech Education and Publishing, London, UK, 2007.

[18] S. Pittaccio, L. Garavaglia, C. Ceriotti, and F. Passaretti, "Applications of shape memory alloys for neurology and neuromuscular rehabilitation," Journal of Functional Biomaterials, vol. 6, no. 2, pp. 328-344, 2015.

[19] Z. Yue, X. Zhang, and J. Wang, "Hand rehabilitation robotics on poststroke motor recovery," Behavioural Neurology, vol. 2017, 2017.

[20] Y. Zhu, The Comparative Study of Flexor and Extensor Alternation of Functional Electrical Stimulation and EA with Stroke Grip Function, Heilongjiang University of Chinese Medicine, Harbin, China, 2011.

[21] J. A. Shaw and C. B. Churchill, "Tips and tricks for characterizing shape memory alloy wire: Part 1-differential scanning calorimetry and basic phenomena," Experimental Techniques, vol. 2008, 2008.

[22] S. Szykowny and M. H. Elahinia, "Heat transfer analysis of shape memory alloy actuators," 2006.

[23] B. Zhou, Y. J. Liu, J. S. Leng et al., "The constitutive models of shape memory alloys," Science in China G, vol. 39, no. 7, pp. 998-1006, 2009.

[24] P. C. C. Monteiro, L. L. Silva, T. A. Netto et al., "Experimental investigation of the influence of the heating rate in an SMA actuator performance," Sensors and Actuators A: Physical, vol. 199, pp. 254-259, 2012.

[25] Y.-J. Lai, L.-J. Yeh, and M.-C. Chiu, “An experimental investigation on shape memory alloy dynamic splint for a finger joint application," Sensors and Actuators A: Physical, vol. 173, no. 1, pp. 210-218, 2012.

[26] S. Bigger and W. Yao, "Design and evaluation of a soft and wearable robotic glove for hand rehabilitation," IEEE
Transactions on Neural Systems and Rehabilitation Engineering, vol. 24, no. 10, 2016.

[27] W. R. Chen, Research on Theory and Methodology of Underactuated Anthropomorphic Hand Design for Grasping and Manipulation, Huazhong University of Science and Technology, Wuhan, China, 2017.

[28] E. B. Brokaw, I. Black, R. J. Holley, and P. S. Lum, "Hand spring operated movement enhancer (HandSOME): a portable, passive hand exoskeleton for stroke rehabilitation," IEEE Transactions on Neural Systems and Rehabilitation Engineering, vol. 19, no. 4, pp. 391-399, 2011. 